\title{
Universal Neonatal Hearing Screening NEONATOLOGIST'S PERSPECTIVE
}

\author{
AdITYa RaKHECHa \\ Department of Neonatology, St Mary's Hospital, Central Manchester University Hospitals, Manchester, UK. \\ arakhecha@hotmail.com
}

$\mathrm{P}$ ermanent hearing loss is one of the commonest congenital disorders with the incidence being much more than the conditions newborns are routinely screened for. Most neonatal hearing loss is sensorineural and a known genetic cause can be found in only $50 \%$. Universal Neonatal Hearing Screening (UNHS) is restricted to developed countries due to cost of delivery and manpower required in screening. It has been conclusively shown to significantly lower the age of diagnosis of children with hearing loss [1]. Targeted screening of high risk infants would miss $50 \%$ of babies that would have been identified by UNHS [2]. In the absence of a screening program, hearing loss is typically identified with language delay around 24 months of age in contrast to three months or younger in the screened population with intervention by six months. Screening has reduced the age, at which infants receive hearing aids, from 13-16 months to 5-7 months in developed countries [3].

Auditory stimuli during the first 6 months of life are critical for the development of speech and language skills. Several studies have shown that infants who receive intervention before the age of 6 months have better school outcomes, and improved language and communication skills by ages 2 to 5 years [4]. Without early intervention, children with hearing loss will show irreversible deficit in communication, psychosocial skills and literacy. They are more likely to have academic underachievement, problems with employment and psychological distress.

Screening can be performed by otoacoustic emissions $(\mathrm{OAE})$ or automated auditory brainstem response (AABR) testing. OAE is technically easier and faster to perform. It is cheaper but has higher false positive rates of about $15 \%$. It also requires a quite or a soundproof room. In comparison, $\mathrm{AABR}$ has less false positives and can also detect patients with auditory neuropathy unlike OAE. It is best to screen after 24 hours as pass rate increase from $70 \%$ to $82 \%$ if done after 24 hours [5]. This may be attributed to obstruction of ear canal with vernix, debris and amniotic fluid in the early period.

There are limitations with hearing screening. UNHS will not identify progressive and late onset hearing loss as well as less severe hearing loss $(<40 \mathrm{~dB})$. The false positive rate is around $2 \%$ that is similar to thyroid screening. This can cause anxiety in parents [6]. An effective program needs a large amount of organization and should have an integrated diagnosis, intervention and follow-up plan. In developed world where the state pays for health care, the benefits of UNHS outweigh the costs. However, the cost effectiveness has not been established in developing countries.

The study in this issue by Augustine, et al. [7] used BERAphone (AABR) for screening the infants. They found that the BERAphones were easy to use and worked well even in high ambient noise. First screening was achieved in $97.7 \%$ infants that is above $95 \%$ recommended by the Joint Committee on Infant Hearing (JCIH) [2] suggesting that screening is feasible and effective. Confirmatory testing was done between 1 to 3 months but there was a big drop in the number who attended, with just under one-third undergoing the confirmatory test. This problem has been sighted in other programs, including those in the developed countries. Studies have shown the main reasons for a drop-out to be: lack of communication with parents, lack of booking of appointments, problems in transportation and lack of understanding of parents about importance of early diagnosis and intervention [8].

The authors have shown that UNHS using BERAphone is feasible. However, the large loss to follow-up is a big hurdle for any screening program to achieve its objective. This is compounded by the fact that babies with previous risk factors are more likely to attend follow-up, making universal hearing screen not much more effective compared to targeted screening. To be really effective, screening needs to be coupled with an early intervention program without which there would be 
no benefit in early diagnosis. In developing countries like India with the high attrition rate in follow-up, the cost effectiveness of UNHS as well as its comparison with targeted screening needs to be evaluated.

Funding: None; Competing interest: None stated.

\section{REFERENCES}

1. Nelson HD, Bougatsos C, Nygren P. Universal newborn Hearing screening: systematic review to update the 2001 US Preventive Services Task Force Recommendation. Pediatrics. 2008;122:e266-76.

2. American Academy of Pediatrics, Joint Committee on Infant Hearing Year 2007 position statement: Principles and guidelines for early hearing detection and intervention programs. Pediatrics. 2007;120:898-921.

3. Canale A, Favero E, Lacilla M, Recchia E, Schindler A, Roggero $\mathrm{N}$, et al. Age at diagnosis of deaf babies: A retrospective analysis highlighting the advantage of newborn hearing screening. Int $\mathrm{J}$ Pediatr Otorhinolaryngol. 2006:70:1283-9.

4. Pimperton H, Kennedy CR. The impact of early identification of permanent childhood hearing impairment on speech and language outcome. Arch Dis Child. 2012;97:648-53.

5. Vohr BR, White KR, Maxon AB, Johnson MJ. Factors affecting the interpretation of transient evoked otoacoustic emission results in neonatal hearing screening. Semin Hearing. 1993;14:57-72.

6. Patel H, Feldman M. Universal newborn screening. Paediatr Child Health. 2001;16:301-5.

7. Augustine AM, Jana AK, Kuruvilla KA, Danda S, Lepcha $\mathrm{A}$, Ebenezer $\mathrm{J}$, et al. Neonatal hearing screening Experience from a tertiary care hospital in Southern India. Indian Pediatr. 2014;51:179-83.

8. Mukhari SZ, Tan KY, Abdullah A. A pilot project on hospital-based universal newborn hearing screening: Lessons learned. Int $\mathrm{J}$ Pediatr Otorhinolaryngol. 2006;70:843-51.

\title{
Neonatal Hearing Screening OTOLARYNGOLOGIST'S PERSPECTIVE
}

\author{
AChal Gulati and Shelly Khanna Chadha \\ Department of ENT and Head and Neck Surgery, Maulana Azad Medical College, New Delhi \\ achalgulati@rediffmail.com
}

$\mathrm{P}$ rograms focused on screening of neonates and infants for hearing loss often need to be led and supervised by otolaryngologists. On the face of it, it seems a simple enough task, that can be well executed with a well-trained team. However, the fact that - this is not as simple as it looks - was brought home to us when we agreed to lead the implementation of the Neonatal Hearing Screening Program in our hospital. Managing such a program requires multiple skills: being able to calm down a baby, interpreting complex audiological tests, counseling the parents of a child who has failed the test, and undertaking data entry and analysis. As specialists in the field of otolaryngology, we are often theoretically aware of the fact that $1-5$ children out of every 1000 live births are affected by hearing loss [1]. The fact that delay in identification of hearing loss can have significant impact on the linguistic and educational outcomes of the child [2], is what motivated us to accept this task.

As clinicians, we are mostly used to being approached by patients and parents who are seeking our advice and expertise; the issue of screening children who are apparently (or mostly) normal can be rather daunting and challenging. The first step of screening begins much before the hearing screener is inserted into the ear. It begins with making the parents aware about the possibility of hearing loss in their child and its consequent impact not only on the child, but also on the family and the society in the current time, and in the future. They need to be informed about the need for the test, the implications of the result and the future course of investigations. Unless this aspect is taken care of, the program would be headed towards failure. Parents may refuse to accept the test. Even when the test has been undertaken, they fail to bring the child for follow-up and often make decisions based on hearsay. Hence, the importance of awareness, providing correct and timely information in a culturally appropriate way and suitable communication strategies cannot be overstated. The other challenge is appointing a team of well-trained and qualified personnel to implement the program. The team must have otolaryngological and audiological professionals as well as pediatricians, nurses, technicians, 